shown reduction in fractional exhaled nitric oxide (FeNO) with increasing body mass index (BMI) however, to our knowledge, no studies have shown this effect when adjusted for confounding variables such as corticosteroid use. We postulate that obesity has an impact on T2 biomarkers independent of confounders.

Methods Data from two recent studies (ID NCT03630432, NCT03858608) was analysed retrospectively, including patient demographics, medical and drug history, BMI, eosinophils, FeNO and atopic status; and most recently available total Immunoglobulin E (IgE) was collected for this analysis. The primary outcome of interest was the effect of BMI on T2 biomarkers: blood eosinophil count, FeNO and total IgE. Multiple linear regression was performed to investigate whether BMI could predict FeNO when adjusted for other variables (age, sex, atopic status, smoking status, allergic rhinitis, perennial rhinitis, and inhaled and oral corticosteroid dose). All statistical analysis was performed using IBM SPSS Statistics (version 27.0.1.0) and significance was set at $<0.05$.

Results The dataset included 102 overweight and obese difficult asthma participants, 26 overweight and obese mild asthma participants and 25 healthy-BMI mild asthma participants (n $=153$ ). In multiple linear regression, BMI was a significant predictor of FeNO ( $\left.\beta=-2.848, p=0.019^{*}\right)$; in this model, for every increase in BMI by $1 \mathrm{~kg} / \mathrm{m}^{2}$, FeNO decreased by 3 parts per billion (ppb) when adjusted for age, sex, atopy, smoking, rhinitis and corticosteroid dose. The overall model was significant, $\mathrm{F}(9,18)=3.20, \mathrm{p}=0.017^{*}$, with an $\mathrm{R}^{2}=$ 0.62 .

Conclusions Our data indicates there is a significant negative relationship between BMI and FeNO after adjusting for relevant variables, particularly corticosteroid use. This could have important implications for endotyping in this already hard-totreat population, and affects subsequent assessment for advanced asthma therapies, such as biologics, that are tailored to T2-high endotypes. Limitations include non-equal weighting between groups and low numbers in this unpowered study.

\section{P209 TREATABLE TRAITS IN DIAGNOSIS-NAÏVE AND UNTREATED PATIENTS WITH SUSPECTED ASTHMA - DATA FROM THE RADICA STUDY}

R Wang, I Choudhury, L Healy, S Drake, L Willmore, J Mitchelle, R Tudge, A Simpson, CS Murray, SJ Fowler. The University of Manchester, Manchester, UK

\subsection{6/thorax-2021-BTSabstracts.318}

Background The identification of treatable traits (TT) has been proposed as a means to facilitate the delivery of precision medicine in severe asthma. We hypothesise that a similar approach may also provide novel insights in symptomatic patients during initial diagnosis, and aimed to evaluate the prevalence of TT in those with and without subsequently-confirmed asthma.

Method Symptomatic yet untreated patients with cliniciansuspected asthma were recruited. Clinical history and examination were carried out before spirometry with bronchodilator reversibility (BDR), fractional exhaled nitric oxide, and bronchial challenges were performed. Blood eosinophils were measured and patients were skin prick tested. An asthma diagnosis was confirmed or refuted following 6-8 weeks of inhaled corticosteroids (ICS). Medication adherence was recorded using INhaler Compliance Assessment (INCA) device.

Results Of 81 adults ( $\geq 16$ years), 12 were excluded as 'unclassifiable' due to borderline results or missing data. Of the remainder, 42 (45\% male, mean [SD] 32.0 [12.3] yrs) were diagnosed with asthma and 27 (25\% male, 37.1 [12.5] yrs) were not. Pulmonary, extrapulmonary and psychosocial TT

Abstract P209 Table 1 Summary of prevalence of treatable traits identified in symptomatic patients

\begin{tabular}{|c|c|c|c|c|}
\hline Category & Treatable traits & Asthma & $\begin{array}{l}\text { Non- } \\
\text { asthma }\end{array}$ & $\mathrm{p}$-value \\
\hline \multirow[t]{12}{*}{ Pulmonary } & Airflow limitation & $12 / 42$ & $0 / 27(0 \%)$ & 0.006 \\
\hline & & $(28.6 \%)$ & & \\
\hline & Bronchodilator reversibility & $19 / 42$ & $0 / 27(0 \%)$ & $<0.001$ \\
\hline & & $(45.2 \%)$ & & \\
\hline & High FeNO ( $\geq 40 \mathrm{ppb})$ & $23 / 42$ & $3 / 27$ & $<0.001$ \\
\hline & & $(54.8 \%)$ & $(11.1 \%)$ & \\
\hline & Blood eosinophilia $\left(\geq 0.4 \times 10^{9}\right.$ & $14 / 40$ & $0 / 25(0 \%)$ & 0.002 \\
\hline & cells/L) & $(35.0 \%)$ & & \\
\hline & Exercise induced breathlessness & $25 / 42$ & $17 / 27$ & 0.974 \\
\hline & & $(59.5 \%)$ & $(63.0 \%)$ & \\
\hline & Bronchial hyperresponsiveness to & $19 / 33$ & $0 / 24(0 \%)$ & $<0.001$ \\
\hline & methacholine & $(57.6 \%)$ & & \\
\hline \multirow{22}{*}{$\begin{array}{l}\text { Extra } \\
\text { pulmonary }\end{array}$} & Symptoms of rhinitis, $\mathrm{n}(\%)$ & $24 / 42$ & $18 / 27$ & 0.590 \\
\hline & & $(57.1 \%)$ & $(66.7 \%)$ & \\
\hline & History of nasal polyps $n(\%)$ & $1 / 42$ & $0 / 27(0 \%)$ & 1.00 \\
\hline & & $(2.4 \%)$ & & \\
\hline & Obesity (BMl>30) & $14 / 42$ & $11 / 27$ & 0.713 \\
\hline & & $(33.3 \%)$ & $(40.7 \%)$ & \\
\hline & Symptoms of reflux & $17 / 42$ & $21 / 27$ & 0.005 \\
\hline & & $(40.5 \%)$ & $(77.8 \%)$ & \\
\hline & Eczema & $12 / 42$ & $3 / 27$ & 0.157 \\
\hline & & $(28.6 \%)$ & $(11.1 \%)$ & \\
\hline & Atopy $^{\infty}$ & $31 / 42$ & $15 / 27$ & 0.191 \\
\hline & & $(73.8 \%)$ & $(55.6 \%)$ & \\
\hline & Cat-sensitised & $6 / 42$ & $5 / 27$ & 0.740 \\
\hline & & $(14.3 \%)$ & $(18.5 \%)$ & \\
\hline & Cat-sensitised and exposure & $2 / 6$ & $1 / 5(20 \%)$ & 1.00 \\
\hline & & $(33.3 \%)$ & & \\
\hline & Dog-sensitised & $4 / 42$ & $0 / 27(0 \%)$ & 0.290 \\
\hline & & $(9.5 \%)$ & & \\
\hline & Dog-sensitised and exposure & $1 / 4$ & $0 / 0$ & $\mathrm{n} / \mathrm{a}$ \\
\hline & & $(25.0 \%)$ & & \\
\hline & Self-reported food allergy & $4 / 42$ & $4 / 27$ & 0.776 \\
\hline & & $(9.5 \%)$ & $(14.8 \%)$ & \\
\hline \multirow[t]{11}{*}{ Psychosocial } & Current smoker & $9 / 42$ & $2 / 27$ & 0.047 \\
\hline & & $(21.4 \%)$ & $(7.7 \%)$ & \\
\hline & Former smoker & $4 / 42$ & $8 / 27$ & \\
\hline & & $(9.5 \%)$ & $(30.7 \%)$ & \\
\hline & Non smoker & $29 / 42$ & $16 / 27$ & \\
\hline & & $(69.0 \%)$ & $(61.5 \%)$ & \\
\hline & Self-reported anxiety/psychiatric & $8 / 42$ & $9 / 27$ & 0.290 \\
\hline & disease & $(19.0 \%)$ & $(33.3 \%)$ & \\
\hline & Medication compliance rate*, & 78.9 & 78.6 & 0.149 \\
\hline & Median (IQR) & $(64.4-$ & $(67.0-$ & \\
\hline & & $89.5) \%$ & $87.0) \%$ & \\
\hline
\end{tabular}

Data presented as $\mathrm{n}(\%), \mathrm{p}$-value calculated using chi-squared test or fisher's exact test FeNO: fractional exhaled nitric oxide; ${ }^{\infty}$ Skin prick test to eight common inhaled aeroallergens. *Data presented as median (IQR), p-value calculated using Mann-Whitney U test. 
were identified in both asthma and non-asthma patients (table 1). Whilst airflow limitation, BDR, airway inflammation and bronchial hyperresponsiveness were more prevalent in asthma than non-asthma, exercise-induced symptoms were equally prevalent in both groups, as were extrapulmonary features such as obesity, rhinitis and atopy. There were more current smokers in asthma (21.4\%) compared to non-asthma $(7.7 \%$, $\mathrm{p}<0.05$ ), but psychosocial history and medication compliance during trial of ICS were similar in both groups. Reflux was the most prevalent TT identified in non-asthma (77.8\%), found almost twice as commonly as in asthma $(40.4 \%$, $\mathrm{p}=0.005)$. Although atopy was the single most common feature in asthma $(73.8 \%)$, the majority of non-asthmatics were also sensitised (55.6\%). Four of 15 (26.7\%, three with asthma) symptomatic and pet-sensitised patients had ongoing pet exposures.

Conclusion Treatable traits are common in patients with symptoms of asthma regardless of diagnostic labels, although the patterns of prominent features may be different, suggesting that personalised management strategy could be offered to symptomatic patients both with and without confirmed asthma. Future research should focus on further characterisation of TT in symptomatic patients with uncertain diagnosis.

\section{Oxygen, CPAP, NIV or ICU: what works in COVID-19?}

\section{P210 NOT ALL COVID-19 DEATHS ARE HYPOXIC: OBSERVATIONAL COHORT STUDY OF PATIENTS WHO DIED AT THE NIGHTINGALE HOSPITAL EXETER}

${ }^{1} \mathrm{KL}$ Adams, ${ }^{2} \mathrm{C}$ Fearnley, ${ }^{2} \mathrm{R}$ Mankiewitz, ${ }^{2} \mathrm{~J}$ Hubbard, ${ }^{2} \mathrm{~W}$ Reed, ${ }^{3} \mathrm{~T}$ Whitehead, ${ }^{2} \mathrm{~L}$ Anning ${ }^{1}$ North Devon District Hospital, Barnstaple, UK; ${ }^{2}$ Torbay and South Devon NHS Foundation Trust, Torbay, UK; ${ }^{3}$ Royal Devon and Exeter NHS Foundation Trust, Exeter, UK

10.1136/thorax-2021-BTSabstracts.319

The Nightingale Hospital Exeter (NHE) with 116 beds designed to deliver respiratory support including non-invasive ventilation and high flow nasal oxygen, managed 242 patients with Covid-19 from November 2020 to February 2021. Patients considered candidates for invasive ventilation were not transferred to NHE. In those who died there were two observed modes of death: those that were hypoxic and patients that died without any significant hypoxia Hypoxic respiratory failure is well-documented as a cause of death in Covid-19 but the mechanism of death in non-hypoxic patients remains incompletely understood.

Methods Electronic patient records were retrospectively reviewed for all deaths at the NHE between 26.11.2020 and 24.02.2021. Data collection included patient characteristics, pre-morbid function and pre-existing respiratory and neurological comorbidities, cause of death at certification, oxygen saturations, Fi02 and level of respiratory support both on admission, last recorded prior to death and the maximum required during admission. Hypoxia was considered significant if the last recorded oxygen saturations prior to death or palliation were $<88 \%$ irrespective of $\mathrm{Fi02}$ or if the patient was receiving $>30 \%$ inspired oxygen.

Results A total of 242 patients from eight NHS Trusts were admitted to the NHE, and $37(15 \%)$ died. Of the patients
Abstract P210 Table 1 Patient demographics of Covid related deaths with and without hypoxia

\begin{tabular}{lll}
\hline & Hypoxic & $\begin{array}{l}\text { Non- } \\
\text { hypoxic }\end{array}$ \\
\hline Age (years) & 84 & 89 \\
Days from positive swab to death (days) & 12 & 19 \\
Pre-existing respiratory disease (total (\%)) & $5(40 \%)$ & $10(42 \%)$ \\
Pre-existing neurological condition (total & $13(52 \%)$ & $7(58 \%)$ \\
$(\%))$ & & $6(50 \%)$ \\
Death attributed to Covid-19 (total (\%)) & $22(88 \%)$ & \\
\hline
\end{tabular}

that died, 32\% had no significant hypoxia. Table 1 compares the two groups. Five patients were treated with high flow nasal oxygen, all of whom died hypoxic. In non-hypoxic patients with an alternative cause of death these were either pneumonia, sepsis or dementia.

Discussion Atypical presentations of Covid-19 in the elderly are well-documented. We observed a population of patients who died after testing positive for Covid-19 who were not hypoxic, but had no other cause of death other than Covid19 identified. Neither underlying respiratory disease nor underlying neurological conditions predicted mode of death although there was a trend towards a hypoxic death in younger patients. Few studies have looked at underlying cause of death from Covid-19 beyond its hypoxic and cardiovascular effects and further research is needed to understand the additional modes of death caused by this virus.

\section{P211 COMPARING OUTCOMES AND CHARACTERISTICS OF COVID-19 PATIENTS TREATED WITH CPAP INSIDE AND OUTSIDE OF THE INTENSIVE CARE UNIT}

DL Sykes, R Martin, MG Crooks, K Thuthu, Ol Brown, TJP Tyrer, A Aji, P Gunasekera, S Faruqi. Hull University Teaching Hospitals NHS Trust, Hull, UK

\subsection{6/thorax-2021-BTSabstracts.320}

Introduction and Objectives Continuous Positive Airway Pressure (CPAP) has been widely adopted to manage hypoxaemic respiratory failure secondary to COVID-19 pneumonia. Currently promising data is available to suggest the success this as a definitive treatment for such patients, both inside and outside of the ICU. We aim to compare the outcomes and characteristics of patients treated with CPAP in these two settings. Methods In this single-centre prospective observational study we observed the baseline characteristics, physiological observations, laboratory tests, and outcomes of all consecutive patients with COVID-19 pneumonia between April 2020 and March 2021 treated with CPAP. We report data from patients treated in both the ICU and the designated COVID-19 Respiratory Support Unit.

Results We report the outcomes of 187 patients (Mean Age $=66.1[\mathrm{SD}=12.7], 64 \%$ Male $[\mathrm{n}=120])$ with COVID-19 pneumonia treated with CPAP in a single NHS trust. Overall mortality for these patients was $45 \%(n=84)$, this was significantly higher for the patients treated outside of the ICU $(29 \%$ vs $60 \%, \mathrm{p}<0.001)$. Those who received CPAP in the ICU were significantly younger than those treated outside of this setting (Mean Age $(\mathrm{SD})=61.2$ (13.9) vs. 70.9 (11.8), $\mathrm{p}<0.001)$. The group of patients treated outside of 